\title{
Relação vitamina E:vitamina C sobre a qualidade da carne de frangos submetidos ao estresse pré-abate
}

\author{
[Vitamin E:Vitamin C relationship on meat quality of broiler chicken submitted \\ to pre-slaughter stress] \\ J.I.M. Fernandes ${ }^{1}$, M.I. Sakamoto ${ }^{2}$, D.C. Peiter ${ }^{1}$, E.T. Gottardo ${ }^{1}$, C. Tellini ${ }^{1}$ \\ ${ }^{1}$ Universidade Federal do Paraná - Palotina, PR \\ ${ }^{2}$ Universidade Camilo Castelo Branco - Descalvado, SP
}

\begin{abstract}
RESUMO
Determinou-se a melhor relação vitamina E:vitamina $\mathrm{C}$ em dietas para frangos de corte, visando ao melhor desempenho produtivo e à melhor qualidade da carne das aves submetidas ao estresse pré-abate. Utilizaram-se 800 pintos de corte, machos, distribuídos em delineamento inteiramente ao acaso, em esquema fatorial $2 \times 4$, com dois níveis de suplementação de vitamina $\mathrm{E}-0$ e $250 \mathrm{mg} / \mathrm{kg}$ - e quatro de vitamina $\mathrm{C}-0,150,300$ e $450 \mathrm{mg} / \mathrm{kg}$. Aos 42 dias de idade, 12 horas ante mortem, amostras de aves de cada tratamento foram submetidas ao estresse por calor e, em seguida, pelo transporte. Foram avaliadas características de desempenho - peso vivo, consumo de ração e conversão alimentar -, bem como rendimento de carcaça e qualidade da carne de peito e coxas - perda de água, cor e $\mathrm{pH}$. Os níveis de vitaminas avaliadas não influenciaram nas características de desempenho avaliadas. Houve menor rendimento de peito $(34,2 v s .34,9 \%)$ e maior $\mathrm{pH}$ inicial $(6,3 \mathrm{vs.} 6,1)$ dos cortes, para aves que sofreram estresse em relação àquelas que não foram submetidas ao estresse pré-abate.
\end{abstract}

Palavras-chave: desempenho, estresse calórico, frangos de corte, perda de água, rendimento de carcaça

\begin{abstract}
The best relationship for vitamin E:vitamin $C$ in diets for broilers regarding growth performance and meat quality of birds submitted to pre-slaughter stress was determined. 800 male chicks at one day of age were distributed in a completely randomized design in a $2 \times 4$ factorial scheme, with two levels of vitamin E supplementation - 0 and $250 \mathrm{mg} / \mathrm{kg}$ - and four of vitamin C-0, 150, 300 and $450 \mathrm{mg} / \mathrm{kg}$. At 42 days of age, 12 hours ante-mortem, samples of birds from each treatment were submitted to heat stress and then transportation. The performance characteristics evaluated were body weight, feed intake and feed:gain ratio, carcass yield and meat quality of breast and thighs, water loss, color and $\mathrm{pH}$. The levels of vitamins evaluated did not influence the performance characteristics measured. There was a lower breast yield (34.2 vs 34.9\%) and higher initial pH (6.3 vs 6.1) in the cuts for birds that suffered stress than for those who did not undergo pre-slaughter stress.
\end{abstract}

Key words: broiler chickens, carcass yield, heat stress, performance, water loss

\section{INTRODUÇÃO}

A cadeia da carne de frango no Brasil é um importante setor do agronegócio, ocupando a terceira maior produção mundial e o primeiro lugar nas exportações. Para atender a demanda, é fundamental minimizar os problemas de manejo que causam problemas fisiológicos, os quais prejudicam o desempenho produtivo das aves. $\mathrm{O}$ manejo pré-abate e o estresse durante a criação são considerados fatores negativos na produção avícola, considerando-se a seleção genética intensiva dos frangos na obtenção de maior crescimento em menor tempo de criação, o que pode reduzir a taxa de crescimento das aves e aumentar a mortalidade, prejudicando, assim, o rendimento e a qualidade da carne de frango (Savenije et al., 2002).

Recebido em 10 de agosto de 2011 
Aves normalmente sintetizam a vitamina $\mathrm{C}$ e não há necessidade de suplementação dessa vitamina na alimentação. No entanto, sob condições de estresse, a suplementação de vitamina $\mathrm{C}$ pela água de beber ou pela ração tem demonstrado, em alguns casos, aliviar os efeitos deletérios dos fatores de estresse (Silva et al., 1993).

De acordo com a revisão de literatura referente à utilização do ácido ascórbico na avicultura, observam-se alterações nas condições fisiológicas, nas quais a vitamina $\mathrm{C}$ atua como agente antiestresse, com redução das taxas de glicocorticoides, o que resulta em diminuição da degradação tissular, permitindo, assim, maior ganho de peso das aves após um período de estresse (Pardue e Thaxton, 1986). Da mesma forma, a vitamina $\mathrm{E}$ tem sido estudada na suplementação de dietas para as aves, acima do nível recomendado pelo NRC (National..., 1994), que é de $12 \mathrm{mg} / \mathrm{kg}$ de ração para frangos de corte, por apresentar função antioxidante no sistema biológico, além das funções reprodutivas e imunológicas (Colnago et al., 1984; Rice e Kennedy, 1988; Finch e Turner, 1996).

Para conservar sua eficácia, a vitamina $\mathrm{E}$ requer a presença da vitamina $\mathrm{C}$, que torna possível sua regeneração. A vitamina $\mathrm{C}$ é um antioxidante hidrossolúvel, a qual remove os radicais superóxido hidroxila e oxigênio, antes que atinjam os lipídeos celulares e iniciem a peroxidação, além de preservar os níveis de vitamina $E$ e $\beta$-caroteno, antioxidantes endógenos na LDL, durante o estresse oxidativo (Araújo et al., 2010).

Um dos maiores problemas na comercialização da carne de frangos tem sido a rancidez oxidativa, tornando o produto inaceitável do ponto de vista sensorial pelos consumidores. A vitamina $\mathrm{E}$ pode agir sobre essa oxidação aumentando a estabilidade devido ao grau de insaturação da gordura, o que melhora a resistência das carnes frescas e dos produtos cárneos à oxidação (Lauridsen et al., 1997). Cortinas et al. (2005) concluíram que a suplementação com até $200 \mathrm{mg} / \mathrm{kg}$ de a-tocoferol previne até $88 \%$ da máxima oxidação lipídica, no entanto níveis superiores a este não apresentaram melhora na estabilidade da carne de coxas de frangos. Segundo Leeson (2007), geralmente a suplementação de 100-400UI de vitamina E/kg de dieta tem demonstrado promover a qualidade da carne na maioria dos estudos.

Sendo assim, o objetivo deste trabalho foi avaliar as vitamina $\mathrm{E}$ e $\mathrm{C}$ sobre o desempenho e a qualidade da carne de frangos de corte, submetidos ao estresse pré-abate de temperatura e transporte.

\section{MATERIAL E MÉTODOS}

O experimento foi aprovado pelo Comitê de Conduta Ética no Uso de Animais em Experimentação da Universidade Federal do Paraná, Campus Palotina, sob protocolo no.13/2010, em 14 de março de 2010. Foram utilizados 800 pintos de corte, Ross, machos, distribuídos em delineamento inteiramente ao acaso, em esquema fatorial $2 \times 4$, com dois níveis de suplementação de vitamina $E$ - 0 e $250 \mathrm{mg} / \mathrm{kg}$ - e quatro de vitamina $\mathrm{C}-0,150,300 \mathrm{e}$ $450 \mathrm{mg} / \mathrm{kg}$ de ração -, totalizando oito tratamentos com quatro repetições de 25 aves cada.

As rações foram à base de milho e farelo de soja, de acordo com as exigências nutricionais de Rostagno et al. (2005) (Tab. 1). O suplemento vitamínico utilizado foi isento das vitaminas em estudo, e as vitaminas C (protegida) e E foram adicionadas à ração-controle em substituição ao inerte, de acordo com os tratamentos.

As características de desempenho avaliadas, semanalmente, foram: peso vivo, consumo de ração e conversão alimentar. Aos 42 dias de idade, 12 horas ante mortem, oito aves/tratamento foram submetidas ao estresse por calor e, em seguida, pelo transporte. Estas aves foram identificadas por anilhas e mantidas em ambiente com temperatura média de $32^{\circ} \mathrm{C}$ durante $\mathrm{o}$ período ante mortem. Por aproximadamente 30 minutos antes do abate, as aves foram colocadas em caixas de grades plásticas e transportadas em veículo por terreno acidentado e abatidas em estado de ofegação. Outras oito aves/tratamento foram mantidas e transportadas em condições de conforto térmico (temperatura média de $22^{\circ} \mathrm{C}$ ). Após o abate, foram avaliados o rendimento de carcaça e cortes (peito e coxas) e as características de qualidade da carne (perda de água, cor e pH). A determinação do $\mathrm{pH}$ foi realizada diretamente no filé do peito das aves utilizadas para o 


\section{Fernandes et al.}

rendimento de carcaça, com auxílio do potenciômetro Sentron 1001, aos 30 minutos e 24 horas post mortem. O ponto de incisão do eletrodo foi na parte cranial ventral do filé, conforme descrito por Boulianne e King (1995).

As medidas de cor foram realizadas na face ventral do filé $24 \mathrm{~h}$ post mortem, tomando três pontos diferentes de leitura por amostra para cada sistema de cor, nas mesmas amostras da determinação de $\mathrm{pH}$, sendo utilizado o colorímetro Minolta CR10. A análise da cor foi realizada pelo sistema Hunter, considerando-se apenas o valor de L* (luminosidade).

As medidas de perda de água foram realizadas conforme o método descrito por Barbut (1996).
Neste procedimento, $5 \mathrm{~g}$ de amostras, em duplicata, no tempo de $24 \mathrm{~h}$ post mortem, foram homogeneizadas com $8,0 \mathrm{~mL}$ de solução de $\mathrm{NaCl}$ 0,6M. O homogenato foi transferido para tubo de centrífuga de policarbonato e mantido em banho de gelo fundente por 30 minutos e, então, centrifugado a $7000 \mathrm{~g}$ por cinco minutos a $20^{\circ} \mathrm{C}$.

Posteriormente, o sobrenadante foi descartado, e os tubos pesados em balança analítica. Os resultados foram expressos como o peso da solução salina retida.

Os resultados obtidos foram submetidos à análise de variância, e as médias comparadas pelo teste Tukey, ao nível de $5 \%$ de significância, utilizando-se o programa estatístico SAS (Statistical..., 2002).

Tabela 1. Composição percentual e calculada das dietas experimentais dos frangos de corte no período inicial (um a 21 dias) e crescimento (22 a 42 dias)

\begin{tabular}{|c|c|c|}
\hline Ingrediente $(\%)$ & Dieta inicial & Dieta crescimento \\
\hline Milho & 52,68 & 61,23 \\
\hline Farelo de soja & 39,34 & 30,06 \\
\hline Óleo de soja & 3,14 & 3,97 \\
\hline Calcário & 0,98 & 1,02 \\
\hline Fosfato bicálcico & 2,09 & 1,98 \\
\hline Inerte (Caulim) & 0,50 & 0,50 \\
\hline Sal comum & 0,37 & 0,36 \\
\hline Bicarbonato de sódio & 0,09 & 0,11 \\
\hline L-Lisina $\mathrm{HCl} 78 \%$ & 0,18 & 0,21 \\
\hline DL-Metionina $98 \%$ & 0,29 & 0,22 \\
\hline L-Treonina $98 \%$ & 0,04 & 0,04 \\
\hline Premix vitamínico e mineral ${ }^{1,2}$ & 0,30 & 0,30 \\
\hline \multicolumn{3}{|l|}{ Valor calculado } \\
\hline Proteína bruta, $\%$ & 22,50 & 19,00 \\
\hline Energia metabolizável, kcal/kg & 2.949 & 3.100 \\
\hline Cálcio, \% & 1,00 & 0,97 \\
\hline Fósforo disponível, \% & 0,50 & 0,47 \\
\hline Sódio, \% & 0,19 & 0,19 \\
\hline Potássio, \% & 0,87 & 0,72 \\
\hline Cloro, $\%$ & 0,30 & 0,30 \\
\hline Lisina digestível, \% & 1,25 & 1,06 \\
\hline AAST digestível, \% & 0,89 & 0,75 \\
\hline Treonina digestível, $\%$ & 0,80 & 0,68 \\
\hline Arginina digestível, \% & 1,45 & 1,18 \\
\hline $\mathrm{BED}^{3}, \mathrm{mEq} / 100 \mathrm{~g}$ & 228,22 & 192,72 \\
\hline \multicolumn{3}{|c|}{$\begin{array}{l}{ }^{\mathrm{T}} \text { Mistura vitamínica (conteúdo por kg de premix): vit. A 7.000.000,00 UI; vit. D3 } 2.200 .000,00 \mathrm{UI} \text {; vit. K3 1.600,00mg; vit. B1 } \\
2.000,00 \mathrm{mg} \text {; vit. B2 } 5.000,00 \mathrm{mg} \text {, vit. B12 } 12.000,00 \mathrm{mg} \text {; niacina } 35.000,00 \mathrm{mg} \text {; ácido pantotênico } 13.000,00 \mathrm{mg} \text {; ácido fólico } \\
800,00 \mathrm{mg} \text {; antioxidante } 100.000,00 \text {; veículo q.s.p. } 1.000,00 \mathrm{~g} \text {. } \\
{ }^{2} \text { Mistura mineral (conteúdo por kg de premix): ferro } 10.000,00 \mathrm{mg} \text {; cobre } 16.000,00 \mathrm{mg} \text {; iodo } 2.400,00 \mathrm{mg} \text {; zinco } 100.000,00 \mathrm{mg} \text {; } \\
\text { manganês } 140.000,00 \mathrm{mg} \text {; selênio 400,00mg; veículo q.s.p. } 1.000,00 \mathrm{~g} \text {. } \\
{ }^{3} \text { Balanço eletrolítico da dieta (Mongin,1981): BED=[(\% Na*10.000/22,990)+(\% K*10.000/39,102)]-(\% Cl*10.000/35,453). }\end{array}$} \\
\hline
\end{tabular}




\section{RESULTADOS E DISCUSSÃO}

Os valores médios das características de desempenho estão apresentados na Tab. 2. As vitaminas $\mathrm{C}$ e $\mathrm{E}$ avaliadas não influenciaram o ganho de peso, o consumo de ração e a conversão alimentar dos frangos de corte durante os 42 dias de criação.

Estes resultados estão de acordo com Cardoso et al. (2007), que avaliaram a associação da vitamina $\mathrm{E}-0,12$ e $120 \mathrm{mg} / \mathrm{kg}$ - e zinco - 0, 40 e $400 \mathrm{mg} / \mathrm{kg}$ - em dietas para frangos de corte, e não observaram efeito da vitamina $\mathrm{E}$ sobre o ganho de peso, o consumo de ração e a conversão alimentar das aves. Frigg et al. (1992) e Sell et al. (1994), ao utilizarem 300 e 500mg de vitamina $\mathrm{E} / \mathrm{kg}$ da dieta, respectivamente, não verificaram efeito dos tratamentos sobre $\mathrm{o}$ desempenho de frangos de corte e perus. Toledo et al. (2006), ao utilizarem 10, 20 e $30 \mathrm{mg}$ de vitamina $\mathrm{E} / \mathrm{kg}$ da dieta, abaixo dos valores médios utilizados na indústria avícola, não observaram diferença no desempenho dos frangos, o que poderia levar à diminuição nos custos de produção. Resultados semelhantes foram apresentados por Almeida et al. (2009), que não encontraram diferença no ganho de peso e na conversão alimentar de frangos de corte criados até 49 dias de idade com ração suplementada até $400 \mathrm{mg}$ de vitamina $\mathrm{E} / \mathrm{kg}$ da dieta.

Tabela 2. Ganho de peso (GP), consumo de ração (CR) e conversão alimentar (CA) dos frangos de corte suplementados com vitaminas $\mathrm{C}$ e $\mathrm{E}$

\begin{tabular}{|c|c|c|c|c|c|c|c|c|c|}
\hline & GP $(g)$ & CR (g) & CA & GP $(g)$ & CR (g) & CA & GP (g) & CR (g) & CA \\
\hline & \multicolumn{3}{|c|}{1 a 21 dias } & \multicolumn{3}{|c|}{21 a 42 dias } & \multicolumn{3}{|c|}{1 a 42 dias } \\
\hline \multicolumn{10}{|c|}{ Vit. C (mg/kg) } \\
\hline 0 & 728,25 & 1046,75 & 1,437 & 1742,81 & 3289,71 & 1,888 & 2471,05 & 4336,22 & 1,755 \\
\hline 150 & 747,25 & 1068,99 & 1,431 & 1708,45 & 3257,00 & 1,906 & 2455,70 & 4321,51 & 1,760 \\
\hline 300 & 756,41 & 1076,69 & 1,423 & 1720,34 & 3299,97 & 1,918 & 2476,75 & 4375,23 & 1,767 \\
\hline 450 & 748,79 & 1063,26 & 1,420 & 1742,32 & 3335,40 & 1,914 & 2491,11 & 4397,27 & 1,765 \\
\hline \multicolumn{10}{|c|}{ Vit. E (mg/kg) } \\
\hline 0 & 739,01 & 1063,89 & 1,440 & 1746,75 & 3322,64 & 1,902 & 2485,76 & 4384,76 & 1,764 \\
\hline 250 & 751,51 & 1063,97 & 1,416 & 1708,07 & 3263,90 & 1,911 & 2459,58 & 4325,90 & 1,759 \\
\hline Média & 745,20 & 1063,99 & 1,427 & 1728,12 & 3294,84 & 1,906 & 2473,32 & 4356,78 & 1,761 \\
\hline $\mathrm{CV}(\%)$ & 5,97 & 7,64 & 5,80 & 5,63 & 4,90 & 6,60 & 2,57 & 3,35 & 4,34 \\
\hline \multicolumn{10}{|c|}{ Análise de variância } \\
\hline Vit. C & NS & NS & NS & NS & NS & NS & NS & NS & NS \\
\hline Vit. E & NS & NS & NS & NS & NS & NS & NS & NS & NS \\
\hline Vit $\mathrm{C} \times \mathrm{E}$ & NS & NS & NS & NS & NS & NS & NS & NS & NS \\
\hline
\end{tabular}

Valores seguidos por letras diferentes na mesma linha diferem entre si pelo teste de Tukey $(\mathrm{P}<0,05)$. NS $=$ não significativo $(\mathrm{P}>0,05)$

Avaliando o desempenho de frangos de corte até o $49^{\circ}$ dia de idade, alimentados com dieta abaixo da exigência proposta pelo NRC (National..., 1994) para a vitamina $\mathrm{E}$, que é de $12 \mathrm{mg} / \mathrm{kg}$ de dieta, ou ainda com valor considerado excessivo, $300 \mathrm{mg} / \mathrm{kg}$, Raza et al. (1997) verificaram melhor conversão alimentar e maior peso corporal para as aves alimentadas com $300 \mathrm{mg} / \mathrm{kg}$ da dieta. Da mesma forma, Barreto et al. (1999) demonstraram que o aumento da vitamina $\mathrm{E}$ na dieta de frangos de corte resultou em melhora significativa no ganho de peso das aves aos 42 dias de idade.
Silva et al. (1993) observaram melhora na conversão alimentar dos frangos, dos 35 aos 48 dias de idade, que receberam $75 \mathrm{mg}$ de vitamina $\mathrm{C} / \mathrm{kg}$ de dieta e mantidos sob estresse de lotação, em relação às aves que não receberam suplementação da vitamina.

Não houve efeito significativo das vitaminas $\mathrm{C}$ e E sobre o rendimento de carcaça e características de qualidade da carne avaliadas. No entanto, foi observado efeito do estresse pré-abate sobre o rendimento de peito e $\mathrm{pH}$ inicial dos cortes (Tab. 3). Houve menor rendimento de peito para aves que sofreram estresse em relação àquelas 


\section{Fernandes et al.}

que não foram submetidas ao estresse pré-abate. $\mathrm{O}$ pH inicial da carne do peito das aves que sofreram estresse foi maior que das aves não estressadas.

Animais submetidos às condições estressantes têm seu metabolismo acelerado, devido à liberação desordenada de cálcio, levando a um aumento na temperatura corporal (Lesiów e Kijowski, 2003). Para as aves manterem a temperatura interna do corpo relativamente constante, ativam processos metabólicos responsáveis por compensações fisiológicas. Esses ajustes promovem o consumo de nutrientes para produzir ou dissipar calor, ao invés de utilizá-lo para a síntese de novas moléculas (Tinôco, 2001). Tais alterações metabólicas aumentam a produção de ácido láctico e a rigidez muscular (Owens et al, 2000; Lesiów e Kijowski, 2003).

Tabela 3. Rendimento de carcaça e características de qualidade da carne de frangos de corte suplementados com vitaminas $\mathrm{C}$ e E, submetidos ao estresse pré-abate

\begin{tabular}{|c|c|c|c|c|c|c|c|c|c|c|c|c|c|c|}
\hline & \multirow{2}{*}{\multicolumn{2}{|c|}{$\begin{array}{c}\text { Carcaça } \\
(\%)\end{array}$}} & \multicolumn{2}{|c|}{$\begin{array}{c}\text { Peito } \\
(\%)\end{array}$} & \multicolumn{2}{|c|}{$\begin{array}{c}\text { Coxas } \\
(\%)\end{array}$} & \multicolumn{2}{|c|}{$\begin{array}{l}\text { Perda de } \\
\text { água }(\%)\end{array}$} & \multicolumn{2}{|c|}{ Cor (L*) } & \multicolumn{2}{|c|}{$\mathrm{pH}$ inicial } & \multicolumn{2}{|c|}{$\mathrm{pH}$ final } \\
\hline & & & \multicolumn{2}{|c|}{ Estresse } & \multicolumn{2}{|c|}{ Estresse } & \multicolumn{2}{|c|}{ Estresse } & \multicolumn{2}{|c|}{ Estresse } & \multicolumn{2}{|c|}{ Estresse } & \multicolumn{2}{|c|}{ Estresse } \\
\hline & sem & com & sem & com & sem & com & sem & Com & sem & com & sem & com & sem & com \\
\hline \multicolumn{15}{|c|}{ Vit. C (mg/kg) } \\
\hline 0 & 74,6 & 75,5 & 33,2 & 33,7 & 27,6 & 28,1 & 30,2 & 33,2 & 54,7 & 51,5 & 6,1 & 6,3 & 5,9 & 6,0 \\
\hline 150 & 74,9 & 74,9 & 35,1 & 33,9 & 26,8 & 27,3 & 27,0 & 28,7 & 52,9 & 52,7 & 6,2 & 6,2 & 5,9 & 5,9 \\
\hline 300 & 74,3 & 72,7 & 36,3 & 34,0 & 27,3 & 27,6 & 26,2 & 32,2 & 55,8 & 52,1 & 6,1 & 6,3 & 5,8 & 5,9 \\
\hline 450 & 73,5 & 74,1 & 35,1 & 35,2 & 27,9 & 27,5 & 33,0 & 31,7 & 53,2 & 54,8 & 6,2 & 6,3 & 5,9 & 5,9 \\
\hline \multicolumn{15}{|c|}{ Vit. E (mg/kg) } \\
\hline 0 & 74,8 & 73,9 & 34,1 & 35,7 & 27,2 & 27,6 & 29,0 & 31,7 & 54,5 & 53,8 & 6,2 & 6,3 & 5,9 & 5,9 \\
\hline 250 & 75,2 & 73,4 & 33,8 & 34,6 & 27,7 & 27,5 & 29,2 & 31,2 & 53,9 & 52,1 & 6,1 & 6,3 & 5,9 & 5,9 \\
\hline Média & 74,5 & 74,1 & $34,9 \mathrm{a}$ & $34,2 b$ & 27,4 & 27,6 & 29,1 & 31,5 & 54,2 & 52,9 & $6,1 b$ & $6,3 a$ & 5,9 & 5,9 \\
\hline $\mathrm{CV}(\%)$ & \multicolumn{2}{|c|}{4,98} & \multicolumn{2}{|c|}{6,67} & \multicolumn{2}{|c|}{6,16} & \multicolumn{2}{|c|}{23,25} & \multicolumn{2}{|c|}{5,77} & \multicolumn{2}{|c|}{2,48} & \multicolumn{2}{|c|}{2,12} \\
\hline \multicolumn{15}{|c|}{ Análise de variância } \\
\hline Vit. C & \multirow{2}{*}{\multicolumn{2}{|c|}{$\begin{array}{l}\text { NS } \\
\text { NS }\end{array}$}} & \multicolumn{2}{|c|}{ NS } & \multicolumn{2}{|c|}{ NS } & \multicolumn{2}{|c|}{ NS } & \multicolumn{2}{|c|}{ NS } & \multicolumn{2}{|c|}{ NS } & \multicolumn{2}{|c|}{ NS } \\
\hline Vit. E & & & \multirow{2}{*}{\multicolumn{2}{|c|}{ NS }} & \multicolumn{2}{|c|}{ NS } & & S & $\mathrm{N}$ & S & & & & \\
\hline $\begin{array}{l}\text { Vit.Cx } \\
\text { E }\end{array}$ & \multicolumn{2}{|c|}{ NS } & & & & $\mathrm{S}$ & & S & $\mathrm{N}$ & S & & & & \\
\hline Estresse & & & & & & $\mathrm{S}$ & & $\mathrm{S}$ & $\mathrm{N}$ & $\mathrm{S}$ & & & & \\
\hline
\end{tabular}

Valores seguidos por letras diferentes na mesma linha diferem entre si pelo teste de Tukey $(\mathrm{P}<0,05)$; NS= são significativo $(\mathrm{P}>0,05)$.

Souza et al. (2006), ao avaliarem a suplementação de vitamina $\mathrm{E}$ nas dietas de frangos de corte, não observaram efeito dessa vitamina sobre as características produtivas, nos rendimentos de carcaça e cortes, nos valores de $\mathrm{pH}$ e nos teores de lipídeos totais. Foram observados efeitos positivos para força de cisalhamento e concentração de vitamina $\mathrm{E}$ na carne de peito e pernas.

Komiyama et al. (2010) observaram diferença nos valores de $\mathrm{pH}$ em peito de matrizes pesadas quando avaliados em diferentes tempos post mortem (1,5; 4,0 e 24 horas). No entanto, o tempo de jejum, a distância do transporte e o tempo de espera no abatedouro, entre os lotes avaliados, não puderam ser controlados, o que pode ter influenciado nos resultados finais. Segundo alguns autores, essas etapas ante mortem podem afetar a qualidade da carne das aves pelo estresse produzido, levando à alteração no $\mathrm{pH}$ da carne (Takahashi, 2007; Komiyama et al., 2008).

Não foi observado efeito das vitaminas avaliadas e do estresse pré-abate sobre a perda de água do peito das aves ao final do período de criação. Estudos realizados por Roque-Specht et al. (2009), pelo processo de cocção, verificaram maior perda de água, consequentemente, maior perda de peso, em cortes cujos valores de $\mathrm{pH}$ variaram entre 5,2-5,5 e as menores perdas em $\mathrm{pH}$ próximos a 6,0. Este comportamento decorre da hidrólise das proteínas miofibrilares que, em situações de aquecimento e $\mathrm{pH}$ ácido, promove a desnaturação proteica e a perda das propriedades funcionais da carne, diminuindo a capacidade de 
retenção de água (Huff-Lonergan e Lonergan, 2005).

A coloração da carne dos frangos, determinada pela luminosidade $\left(\mathrm{L}^{*}\right)$, não foi influenciada pelas vitaminas avaliadas e pelo estresse préabate nas aves. No entanto, apresentou valores superiores aos considerados normais para filé de peito, que fica em torno de 47,25, de acordo com Komiyama (2006).

Segundo Olivo e Shimokomaki (2001), a presença de vitamina E nas membranas celulares, via suplementação, confere estabilidade de cor e estabilidade lipídica aos cortes cárneos e inibe os processos bioquímicos indutores da carne PSE em frangos de corte.

\section{CONCLUSÃO}

A utilização das vitaminas $\mathrm{E}$ e $\mathrm{C}$ nas dietas de frangos de corte abaixo dos valores médios utilizados na indústria avícola não interferiu no desempenho produtivo das aves. Entretanto, em virtude do elevado estresse pré-abate das aves, a suplementação dessas vitaminas pode minimizar o problema da má qualidade da carne de frangos de corte.

\section{REFERÊNCIAS}

ALMEIDA, A.P.S.; PINTO, M.F.; POLONI, L.B. et al. Efeito do consumo de óleo de linhaça e de vitamina E no desempenho e nas características de carcaças de frangos de corte. Arq. Bras. Med. Vet. Zootec., v.61, p.698-705, 2009.

ARAÚJO, W.A.G.; ROSTAGNO, H.S.; ALBINO, L.F.T. et al. Vitamina E na nutrição animal. Rev. Eletron. Nitritime, v.7, p.12921303, 2010.

BARBUT, S. Estimates and detection of the PSE problem in young turkey breast meat, Can. J. An. Sci., v.76, p.455-457, 1996.

BARRETO, S.L.T.; FERREIRA, W.M.; MORAES, T. Efeito de níveis de vitamina E na dieta sobre o desempenho e concentração de atocoferol na carne de frangos de corte. Arq. Bras. Med. Vet. Zootec., v.51, p.387-392, 1999.
BOULIANNE, M.; KING, A.J. Biochemical and color characteristics of skinless boneless pale chicken breast. Poult. Sci., v.74, p.1693-1698, 1995.

CARDOSO, A.L.S.; ALBUQUERQUE, R.; TESSARI, E.N.C. Desempenho de frangos de corte recebendo rações com diferentes níveis de inclusão de zinco e de vitamina E. Arq. Inst. Biol., v.74, p.307-313, 2007.

COLNAGO, G.L.; JENSEN, L.S.; LONG, P.L. Effect of selenium and vitamin $E$ on the development of immunity to coccidiosis in chickens. Poult. Sci., v.63, p.1136-1143, 1984.

CORTINAS, L.; BARROETA, A.; VILLAVERDE, C. et al. Influence of the dietary polyunsaturation level on chicken meat quality: Lipid oxidation. Poult. Sci., v.84, p.48-55, 2005.

FINCH, J.M.; TURNER, R.J. Effect of selenium and vitamin $\mathrm{E}$ on the immune responses of domestic animals. Res. Vet. Sci., v.60, p.97-106, 1996.

FRIGG, M.; WHITEHEAD, C.C.; WEBER, S. Absence of effects of dietary a-tocopherol on egg yolk pigmentation. Br. Poult. Sci., v.33, p.347353, 1992.

HUFF-LONERGAN, E.; LONERGAN, S.M. Mechanisms of water-holding capacity of meat: The role of postmortem biochemical and structural changes. Meat Sci., v.71, p.194-204, 2005.

KOMIYAMA, C.M. Caracterização e ocorrência de carne pálida em frangos de corte e seu efeito na elaboração de produtos industrializados. 2006. 89f. Dissertação (Mestrado em Nutrição e Produção Animal) Faculdade de Medicina Veterinária e Zootecnia, Universidade Estadual Paulista, Botucatu, SP.

KOMIYAMA, C.M.; MENDES, A.A.; TAKAHASHI, S.E. et al. Chicken meat quality as a function of fasting period and water spray. Rev. Bras. Cienc. Avic., v.10, p.179-183, 2008.

KOMIYAMA, C.M.; MENDES, A.A.; SANFELICE, C. et al. Qualidade físico-química e sensorial da carne de peito de matrizes pesadas de descarte. Cienc. Rural, v.40, p.1623-1629, 2010 . 
LAURIDSEN, C.; BUCKEY, D.J.; MORRISEY, P.A. Influence of dietary fat and vitamin $E$ supplementation on $\alpha$-tocopherol levels and fatty acid profiles in chicken muscle membranal fractions and on susceptibility to lipid peroxidation. Meat Sci., v.46, p.9-22, 1997.

LEESON, S. Vitamin requirements: is there basis for reevaluating dietary specifications? World's Poult. Sci. J., v.63, p.255-266, 2007.

LESIÓW, T.; KIJOWSKI, J. Impact of PSE and DFD meat on poultry processing. A review. Pol. J. Food Nutr. Sci., v.12, p.3-8, 2003.

MONGIN, P. Recent advances in dietary cationanion balance: applications. In: POULTRY PROCEEDINGS NUTRITION SOCIETY, 1981, Cambridge. Proceedings... Cambridge: 1981. v.40, p.285-294.

NATIONAL research council. NRC. Nutrient requirements of poultry. 9.ed. Washington, DC: National Academic Press, 1994. 155p.

OLIVO, R.; SHIMOKOMAKI, M. Carnes: No Caminho da Pesquisa. Cocal do Sul: Imprint, 2001. 155p.

OWENS, C.M.; McKEE, S.R.; MATTEWS, N.S. et al. The development of pale, exudative meat in two genetic lines of turkeys subjected to heat stress and its predication by halothane screening. Poult. Sci., v.79, p.430-435, 2000.

PARDUE, S.L.; THAXTON, J.P. Ascorbic acid in poultry: a review. World's Poult. Sci. J., v.42, p.107-123, 1986.

RAZA, F.K.; KHAN, S.A.; RAZA, A. et al. Effect of vitamin $E$ and deficiency and excess on immune system of broiler chickens. Int. J. Anim. Sci., v.12, p.39-41, 1997.

RICE, D.; KENNEDY, S. Vitamin E: function and effects of deficiency. Brit. Vet. J., v.144, p.482-495, 1988.

ROQUE-SPECHT, V.F.; SIMONI, V.; PARISE, N. et al. Avaliação da capacidade de retenção de água em peitos de frango em função do ph final. Rev. Bras. Agrocienc., v.15, p.77-81, 2009.
ROSTAGNO, H.S.; ALBINO, L.F.T.; DONZELE, J.L. et al. Tabelas brasileiras para aves e suínos: Composição de alimentos $e$ exigências nutricionais. 2.ed., Viçosa:UFV, Departamento de Zootecnia, 2005. 186p.

SAVENIJE, B.; LAMBOOIJ, E.; GERRITZEN, M.A. et al. Effects of feed deprivation and transport on preslaughter blood metabolites, early postmortem muscle metabolites, and meat quality. Poult. Sci., v.81, p.699-708, 2002.

SELL, J.; REYNOLDS, D.; JEFFREY, M. Influence of dietary supplementation with vitamin $\mathrm{E}$ and ascorbic acid on vitamin $\mathrm{E}$ status of pouts. Poult. Sci., v.73, p.13, 1994.

SILVA, R.D.M.; MENTEN, J.F.M.; CARDOSO, M.K. Suplementação de vitamina $\mathrm{C}$ associada à densidade de criação no desempenho de frangos de corte. Sci. Agric., v.50, p.490-497, 1993.

SOUZA, P.A.; SOUZA, H.B.A.; PELICANO, E.R.L. et al. Efeito da suplementação de vitamina $\mathrm{E}$ no desempenho e na qualidade da carne de frangos de corte. Rev. Port. Cienc. Vet., v.101, p.87-94, 2006.

STATISTICAL Analysis System - SAS Institute 2002. SAS ${ }^{\circledast}$. User's Guide: Statistics. Version 9.1. 4.ed., Cary. NC. 2002.

TAKAHASHI, S.E. Ocorrência de carne pálida e características de qualidade de carne de frangos de corte. 2007. 86f. Tese (Doutorado em Nutrição e Produção Animal) - Faculdade de Medicina Veterinária e Zootecnia, Universidade Estadual Paulista, Botucatu, SP.

TINÔCO, I.F.F. Avicultura industrial: Novos conceitos de materiais, concepções e técnicas construtivas disponíveis para galpões avícolas brasileiros. Rev. Bras. Cienc. Avic., v.3, p.1-26, 2001.

TOLEDO, G.S.; KLOECKNER, P.; LOPES, J. et al. Níveis das vitaminas A e E em dietas de frangos de corte de 1 a 42 dias de idade. Cienc. Rural, v.36, p.624-629, 2006. 